\title{
Refraction tomography using a waveform-inversion back-propagation technique
}

\author{
Dong-Joo Min ${ }^{1}$ and Changsoo Shin ${ }^{2}$
}

\begin{abstract}
One of the applications of refraction-traveltime tomography is to provide an initial model for waveform inversion and Kirchhoff prestack migration. For such applications, we need a refraction-traveltime tomography method that is robust for complicated and high-velocity-contrast models. Of the many refraction-traveltime tomography methods available, we believe wave-based algorithms to be best suited for dealing with complicated models.

We developed a new wave-based, refraction-tomography algorithm using a damped wave equation and a waveform-inversion back-propagation technique. The imaginary part of a complex angular frequency, which is generally introduced in frequency-domain wave modeling, acts as a damping factor. By choosing an optimal damping factor from the numerical-dispersion relation, we can suppress the wavetrains following the first arrival. The objective function of our algorithm consists of
\end{abstract}

residuals between the respective phases of first arrivals in field data and in forward-modeled data. The model-response, firstarrival phases can be obtained by taking the natural logarithm of damped wavefields at a single frequency low enough to yield unwrapped phases, whereas field-data phases are generated by multiplying picked first-arrival traveltimes by the same angular frequency used to compute model-response phases.

To compute the steepest-descent direction, we apply a waveform-inversion back-propagation algorithm based on the symmetry of the Green's function for the wave equation (i.e., the adjoint state of the wave equation), allowing us to avoid directly computing and saving sensitivities (Fréchet derivatives). From numerical examples of a block-anomaly model and the Marmousi-2 model, we confirm that traveltimes computed from a damped monochromatic wavefield are compatible with those picked from synthetic data, and our refraction-tomography method can provide initial models for Kirchhoff prestack depth migration.

\section{INTRODUCTION}

Refraction tomography has enjoyed widespread use in the delineation of shallow subsurface structures. Refraction tomography has proved to be valuable for obtaining the shallow-structure information needed for static corrections in seismic-reflection data processing. Recently, refraction tomography also was employed to obtain initial velocity models for waveform inversion and Kirchhoff migration, processes that are sensitive to the initial model. Since refraction tomography requires an efficient and accurate method to compute traveltimes and their Fréchet derivatives, there are a variety of refraction-tomography inversion algorithms in the literature.

Early refraction-tomography methods were based on blocky parameterization, which generally suffered from a velocity-depth trade-off. To circumvent this, Hampson and Russell (1984) and
Schneider and Kuo (1985) solved for the weathering thickness by assuming that velocity information is known. Docherty (1992) studied a method of extracting both depth and velocity. Ray-based, refraction-traveltime tomography became feasible for complicated models by applying cell parameterization. In cell-based ray-tracing traveltime tomography, the Fréchet derivative is expressed as the distance measured along a raypath in a velocity cell. White (1989), Zhu and McMechan (1989), and Stefani (1995) described this approach as turning-ray tomography.

Ray-based refraction tomography sometimes encounters an illposed inverse problem, which can be resolved by introducing regularization (see Scales et al., 1990; Zhang and Toksöz, 1998). Raybased traveltime-tomography methods are only valid for smooth media (Zelt and Barton, 1998); moreover, the minimization of an

Manuscript received by the Editor April 8, 2004; revised manuscript received July 22, 2005; published online May 26, 2006; corrected version published online June 2, 2006

${ }_{2}^{1}$ Korea Ocean Research and Development Institute, Ansan, P. O. Box 29, Seoul, 425-600, Korea. E-mail: djmin @kordi.re.kr.

${ }^{2}$ Seoul National University, School of Civil, Urban and Geosystem Engineering, San 56-1, Shillim-dong, Gwanak-Gu, Seoul, 151-742, Korea. E-mail: css@model.snu.ac.kr.

(C) 2006 Society of Exploration Geophysicists. All rights reserved. 
additional damping term, in the case of regularization being applied, penalizes the model roughness.

In order to overcome this weakness in ray-based traveltime tomography, wave-based methods (Woodward and Rocca, 1988; Woodward, 1992; Luo and Schuster, 1990; 1991; Schuster and Quintus-Bosz, 1993) and Fresnel-volume methods (Vasco et al., 1995) have been suggested. Most wave-based traveltime-tomography techniques extract phase differences of first arrivals by applying a time window or a connective function and computing the steepest-descent direction through a back-projection technique. Pyun et al. (2005) proposed using damped monochromatic wavefields for calculating traveltime residuals and explicitly computed Fréchet derivatives using the reciprocity theorem in their refraction-tomography algorithm. Wave-based refraction tomography can give reliable solutions for a complicated and high-velocitycontrast model, but it requires more computational effort than the ray-based method. The Fresnel-volume method, which is a modified ray-tracing method that computes Fresnel volumes along raypaths rather than wavepaths, does not require more computational effort than do the conventional ray-based methods. Although the Fresnel-volume method can be computationally more efficient than the wave-based method, it sometimes fails at low frequencies near the source and receiver (Vasco et al., 1995).

In this study, we suggest a new wave-based refraction-tomography method that extracts first-arrival traveltimes from a damped wavefield at a single frequency and computes the steepest-descent direction by using a back-propagation algorithm. Our method is similar to that of Pyun et al. (2005) in that we take the natural logarithm of the damped monochromatic wavefield $\left[u(\omega)=A(\omega) e^{i \theta(\omega)}\right.$; $A(\omega)$ is the amplitude and $\theta(\omega)$ is the phase] to extract first-arrival phase information, but we do not directly compute Fréchet derivatives to find the steepest-descent direction. To compute the steepest-descent direction, we use a back-propagation algorithm of reverse time migration similar to that used in conventional wavebased tomography techniques. That is, we back-propagate residuals between field data and model responses, then correlate the back-propagated residuals with virtual sources generated by forward modeling, which is based on the adjoint state of the damped wave equation. Lailly (1983) and Tarantola (1984) showed theoretically that waveform inversion was equivalent to migration when applied to reflection data, and the back-propagation algorithm has been commonly used in seismic-waveform inversion (e.g., Gauthier et al., 1986; Pratt et al., 1998) as well as in traveltime tomography (e.g., Luo and Schuster, 1990, 1991; Schuster and Quintus-Bosz, 1993). Among wave-based tomography methods, our method is very similar to the method of Schuster and Quintus-Bosz (1993). The main difference is that Schuster and Quintus-Bosz (1993) extract first-arrival traveltimes by applying a time window to band-limited wavefields, a process that leads to wrapped phases, whereas our method applies a damped wavefield at a frequency low enough to yield unwrapped phases.

In the following sections, we review the relationship between first-arrival traveltime and the strongly damped wavefield, and then we examine the adjoint state of the damped wave equation used in frequency-domain modeling. Next, we introduce our new refraction-tomography technique via reverse-time migration starting from a matrix formalism (the adjoint state) of the wave equation in the frequency domain. Finally, we show numerical examples generated by our tomography algorithm for the blockanomaly model and the Marmousi-2 model. We also present
Kirchhoff migration images obtained from the velocity structure yielded by our refraction-tomography algorithm.

\section{THEORY}

\section{A damped-wave equation}

In the frequency domain, the 2D constant-density acoustic wave equation can be expressed by

$$
-\omega^{2} u=v^{2}\left(\frac{\partial^{2} u}{\partial x^{2}}+\frac{\partial^{2} u}{\partial z^{2}}\right),
$$

where $\omega$ is the angular frequency and $u$ is the pressure or displacement. A discretized finite-difference or finite-element formula of equation 1 can be written as

$$
\mathbf{S u}=\mathbf{f}
$$

with

$$
\mathbf{S}=\mathbf{K}-\omega^{2} \mathbf{M}
$$

where $\mathbf{S}$ is the impedance matrix, $\mathbf{K}$ is the stiffness matrix, $\mathbf{M}$ is the mass matrix, $\mathbf{u}$ is the wavefield vector, and $\mathbf{f}$ is the source vector (Marfurt, 1984). In most cases, the impedance matrix $\mathbf{S}$ is symmetric, which indicates that the wave equation is self-adjoint.

In frequency-domain modeling, we often use the complex angular frequency $\left(\omega^{*}=\omega+i \alpha\right)$ rather than the real angular frequency to suppress wraparound effects (Aki and Richards, 1980; Shin et al., 2003a), and a wavefield with the complex angular frequency can be expressed (Shin et al., 2003a) as

$$
\begin{aligned}
u(x, y, z, \omega) & =A(x, y, z) e^{i \omega^{*} \tau(x, y, z)} \\
& =A(x, y, z) e^{i(\omega+i \alpha) \tau(x, y, z)} \\
& =A(x, y, z) e^{-\alpha \tau(x, y, z)} e^{i \omega \tau(x, y, z)}
\end{aligned}
$$

where $A(x, y, z)$ is the amplitude, $\alpha$ is the damping factor, and $\tau(x, y, z)$ is the traveltime from the source to the receiver or depth. In equation 4, the complex angular frequency's imaginary term acts as a damping term, leading to a damped complex impedance matrix. The damped complex impedance matrix is still symmetric, which guarantees reciprocity and consequently allows us to employ the adjoint state of the damped wave equation. By introducing a damping factor in frequency-domain modeling, we suppress wavetrains following the first arrival (e.g., Shin et al., 2003a). An optimal damping factor can be determined by equation A-1. In practice, however, if we use the damping factor determined by equation A-1, we may encounter numerical overflow. As a result, we must adjust the damping factor in order to avoid numerical overflow within 32-bit double-precision limit.

We illustrate damping effects using the block-anomaly model shown in Figure 1. As indicated, the entire model is $3 \mathrm{~km} \times$ $0.5 \mathrm{~km}$, and the block-anomaly body is $0.4 \mathrm{~km} \times 0.2 \mathrm{~km}$. The velocities of the first layer, the second layer, and the block-anomaly body are $1.5 \mathrm{~km} / \mathrm{s}, 4.5 \mathrm{~km} / \mathrm{s}$, and $3.0 \mathrm{~km} / \mathrm{s}$, respectively. Because we use a grid interval of $15 \mathrm{~m}$, the number of grid points is 201 and 34 along the $x$ - and $z$-axes, respectively. Figures $2 \mathrm{a}$ and $2 \mathrm{~b}$ show 
synthetic seismograms generated without and with damping, respectively. In order to generate synthetic seismograms, we solve the two-way wave equation using the nine-point finite-difference method suggested by Jo et al. (1996). For a damping factor, we choose 83.8 , which is half the value computed by equation A-1. Figure 2a shows multievents, such as direct waves, refracted waves, and reflection waves. Figure $2 \mathrm{~b}$ shows only first arrivals (either direct waves or refracted waves). Figures $2 \mathrm{a}$ and $2 \mathrm{~b}$ are plotted with different gain values. These numerical results show that we can extract first-arrival information from the damped wavefield.

According to Shin et al. (2003a), the first-arrival traveltime can be determined from the imaginary term of the logarithm of the damped monochromatic wavefield expressed as

$\ln u(x, y, z, \omega)=\ln A(x, y, z) e^{-\alpha \tau(x, y, z)}+i \omega \tau(x, y, z)$.

To check the reliability of traveltimes computed from the logarithm of wavefields at a single frequency, we compare numerically computed traveltimes with traveltimes picked on synthetic seismograms (e.g., Figure 2a) in Figure 3. Figure 3 shows that numerically computed traveltimes are comparable to traveltimes picked on synthetic seismograms. There are some discrepancies in traveltimes obtained from approximately $2.8-3.0 \mathrm{~km}$ for the source located at $2.25 \mathrm{~km}$, where refracted waves are not detected by the damped wave equation. The traveltimes are computed at $0.1 \mathrm{~Hz}$, which is a very low frequency. In general, we wish to use a frequency low enough to obtain unwrapped traveltimes (Mora, 1989), and the optimal frequency can be determined by the maximum traveltime of refracted waves (see Appendix B).

\section{Refraction-tomography algorithm}

By exploiting a matrix formalism of the wave equation in the frequency domain, Pratt et al. (1998) determine the steepest-descent direction without explicitly computing the Jacobian matrix, thereby saving computer memory required for storing the Jacobian matrix and computing time. Following Pratt et al. (1998), we implicitly calculate the steepest-descent direction in our refraction tomography. We define the objective function as the $l_{2}$ norm of residuals between phases of field data and model responses at an arbitrarily chosen angular frequency $\omega_{c}$ :

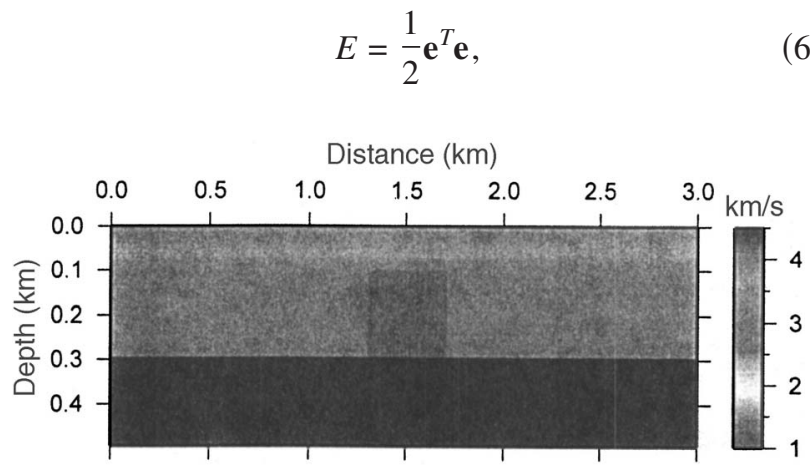

Figure 1 . The geometry of the block-anomaly model.
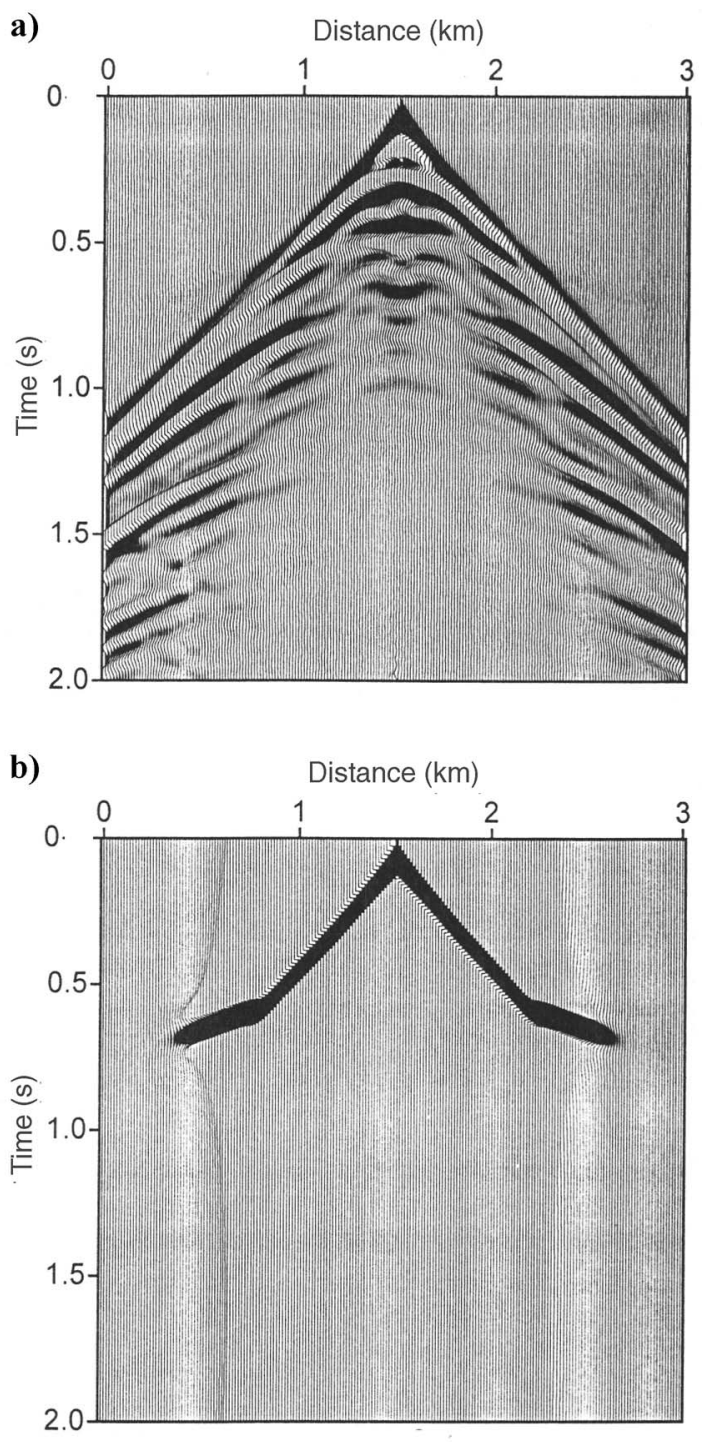

Figure 2. Synthetic seismograms generated by the nine point finitedifference method of Jo et al. (1996) for the block-anomaly model (a) without and (b) with damping factor.

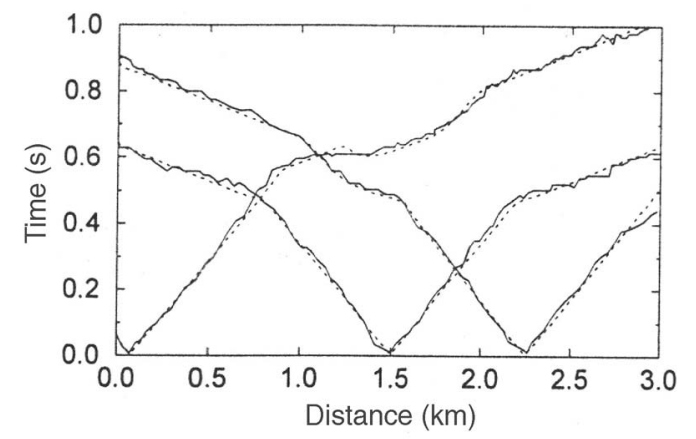

Figure 3. First-arrival traveltimes computed by the $0.1-\mathrm{Hz}$ damped monochromatic wavefield for the block-anomaly model when a source is located at $0.075 \mathrm{~km}, 1.5 \mathrm{~km}$, and $2.25 \mathrm{~km}$. Solid lines indicate traveltimes picked on synthetic seismograms; dotted lines denote traveltimes computed from the damped monochromatic wavefields. 
with

$$
\mathbf{e}=\operatorname{Im}\left\{\ln \frac{\mathbf{d}\left(\omega_{c}\right)}{\mathbf{u}\left(\omega_{c}\right)}\right\}
$$

where Im indicates the imaginary part of a complex number, and $\mathbf{d}\left(\omega_{c}\right)$ and $\mathbf{u}\left(\omega_{c}\right)$ denote field data and model responses, respectively, at an angular frequency. Because we assume that both field data and model responses consist only of first arrivals, their logarithms indicate the phases of their first arrivals. In practice, the phase of the model responses is computed by the logarithm of the damped wavefield, whereas the phase of the field data is obtained by picking first-arrival traveltimes and then multiplying by an angular frequency. The angular frequency is the same one used to compute model responses by the damped wave equation. When we choose the frequency, we favor a frequency low enough to avoid the cycleskipping effect (Shin et al., 2003a). We can express the field data and the model responses as

$$
\begin{aligned}
& d_{i j}\left(\omega_{c}\right)=A_{i j}^{f} e^{i \theta_{i j}^{f}}=A_{i j}^{f} e^{i \omega_{c} t_{i j}^{f},} \\
& u_{i j}\left(\omega_{c}\right)=A_{i j}^{m} e^{i \theta_{i j}^{m}}=A_{i j}^{m} e^{i \omega_{c} t_{i j}^{m},}
\end{aligned}
$$

where the superscripts $m$ and $f$ represent model response and field data, and $i$ and $j$ denote source and receiver numbers, respectively. For simplicity, we assume that receivers are located at all grid points of the surface. The objective function expressed by equations 6 and 7 can be rewritten as

$$
E=\frac{1}{2} \sum_{i=1}^{M} \sum_{j=1}^{N}\left(\theta_{i j}^{f}-\theta_{i j}^{m}\right)^{2}
$$

In the steepest-descent method, it is necessary to compute the gradient of the objective function. By taking the derivative of equation 10 with respect to the $k$ th velocity $v_{k}$ (we divide a $2 \mathrm{D}$ model into $K$ cells, $k=1,2, \ldots, K$ ), we express the gradient of the objective function as

$$
\nabla E_{v_{k}}=-\sum_{i=1}^{M} \sum_{j=1}^{N}\left(\theta_{i j}^{f}-\theta_{i j}^{m}\right) \frac{\partial \theta_{i j}^{m}}{\partial v_{k}},
$$

where the partial-derivative wavefield of the phase with respect to the $k$ th velocity is

$$
\frac{\partial \theta_{i j}^{m}}{\partial v_{k}}=-\operatorname{Im}\left\{\frac{1}{u_{i j}} \frac{\partial u_{i j}}{\partial v_{k}}\right\}
$$

Substituting equation 12 into equation 11 gives

$$
\nabla E_{v_{k}}=\sum_{i=1}^{M} \sum_{j=1}^{N}\left(\theta_{i j}^{f}-\theta_{i j}^{m}\right) \operatorname{Im}\left\{\frac{1}{u_{i j}} \frac{\partial u_{i j}}{\partial v_{k}}\right\} .
$$

To express equation 13 using the model coordinates rather than receiver coordinates, we augment the phase residual vector by zero values and move $1 / u_{i j}$ in equation 13 :

$$
\nabla E_{v_{k}}=\sum_{i=1}^{M} \operatorname{Im}\left\{\left[\begin{array}{lllll}
\frac{\partial u_{i 1}}{\partial v_{k}} \frac{\partial u_{i 2}}{\partial v_{k}} & \cdots & \frac{\partial u_{i N}}{\partial v_{k}} \frac{\partial u_{i(N+1)}}{\partial v_{k}} & \cdots & \frac{\partial u_{i K}}{\partial v_{k}}
\end{array}\right]\left[\begin{array}{c}
\frac{\theta_{i 1}^{f}-\theta_{i 1}^{m}}{u_{i 1}} \\
\frac{\theta_{i 2}^{f}-\theta_{i 2}^{m}}{u_{i 2}} \\
\vdots \\
\frac{\theta_{i N}^{f}-\theta_{i N}^{m}}{u_{i N}} \\
0 \\
\vdots \\
0
\end{array}\right]\right\}
$$

or

$$
\nabla E_{v_{k}}=\sum_{i=1}^{M} \operatorname{Im}\left\{\left[\frac{\partial \mathbf{u}_{i}}{\partial v_{k}}\right] \mathbf{r}_{i}\right\}
$$

with

$$
\mathbf{r}_{i}=\left[\begin{array}{c}
\frac{\theta_{i 1}^{f}-\theta_{i 1}^{m}}{u_{i 1}} \\
\frac{\theta_{i 2}^{f}-\theta_{i 2}^{m}}{u_{i 2}} \\
\vdots \\
\frac{\theta_{i N}^{f}-\theta_{i N}^{m}}{u_{i N}} \\
0 \\
\vdots \\
0
\end{array}\right] .
$$

The partial-derivative wavefield with respect to velocity $\left(\partial \mathbf{u}_{i} /\right.$ $\partial v_{k}$ ) can be computed with the virtual source (Pratt et al., 1998) and the modeling operator from equation 2 . If we take the partial derivative of equation 2 with respect to the $k$ th velocity $v_{k}$, we obtain

$$
\frac{\partial \mathbf{S}}{\partial v_{k}} \mathbf{u}_{i}+\mathbf{S} \frac{\partial \mathbf{u}_{i}}{\partial v_{k}}=0 .
$$

Rearranging equation 17 gives

$$
\frac{\partial \mathbf{u}_{i}}{\partial v_{k}}=\mathbf{S}^{-1} \mathbf{f}_{k, i}^{*}
$$

and

$$
\mathbf{f}_{k, i}^{*}=-\frac{\partial \mathbf{S}}{\partial v_{k}} \mathbf{u}_{i}
$$

We define $\mathbf{f}_{k, i}^{*}$ as the virtual-source vector required to compute the partial derivative of the wavefield with respect to the $k$ th velocity.

By substituting equations 18 and 19 into equation 15, we express the gradient of the objective function with respect to the $k$ th velocity as 


$$
\nabla E_{v_{k}}=\sum_{i=1}^{M} \operatorname{Im}\left\{\mathbf{f}_{k, i}^{* T}\left[\mathbf{S}^{-1}\right]^{T} \mathbf{r}_{i}\right\},
$$

and the total gradient of the objective function is written as

$$
\nabla E=\sum_{i=1}^{M} \operatorname{Im}\left\{\mathbf{F}_{i}^{* T}\left[\mathbf{S}^{-1}\right]^{T} \mathbf{r}_{i}\right\}
$$

given that

$$
\mathbf{F}_{i}^{*}=\left[\begin{array}{lllll}
\mathbf{f}_{1, i}^{*} \mathbf{f}_{2, i}^{*} & \cdots & \mathbf{f}_{k, i}^{*} \cdots & \mathbf{f}_{K, i}^{*}
\end{array}\right]
$$

Equation 21 implies that to compute the steepest-descent direction, we divide the phase differences by the damped wavefields, backpropagate the divided phase differences, and then compute the scalar products between damped back-propagated residuals and damped virtual sources.

In an inversion algorithm using the steepest-descent method, the velocity parameter can be updated by

$$
v^{(n+1)}=v^{(n)}-\beta^{(n)} \nabla E^{(n)},
$$

where $n$ is the iteration number and $\beta^{(n)}$ is the step length, which is chosen to minimize the $l_{2}$ norm in the steepest-descent direction (Pratt et al., 1998). In our algorithm, we use a constant step length, whose value is dependent on model dimensions.

Numerical structure in equation 21 is very similar to that of waveform inversion proposed by Pratt et al. (1998). The main difference between our tomography algorithm and Pratt et al.'s waveform-inversion algorithm is that Pratt et al. back-propagates data residuals at banded frequencies, and we back-propagate only the phase differences divided by damped wavefields at a single frequency.

In Appendix C, we compare our tomography method to that of Schuster and Quintus-Bosz (1993). Schuster and Quintus-Bosz (1993) introduce a time window to extract first arrivals, which results in wrapped phases as shown in equation C-3, whereas we use damped wavefields at a very low frequency, which yields unwrapped phases. Since we use only a single frequency, our method can be more efficient than that of Schuster and Quintus-Bosz (1993).

\section{NUMERICAL EXAMPLES}

Having used the block-anomaly model (Figure 1) to show that traveltimes calculated by damped-wave equations are equivalent to first-arrival traveltimes picked on a synthetic seismogram (e.g., Figures 2 and 3), we use the same model to perform refraction tomography. For field-data traveltime, we employ traveltimes computed by the damped-wave equation for the true block-anomaly model. For the initial model in the inversion algorithm, we use a linearly increasing velocity model, whose velocity ranges from $1.5 \mathrm{~km} / \mathrm{s}$ to $4.5 \mathrm{~km} / \mathrm{s}$ with respect to depth. In Figure 4, we display the initial-velocity model, the velocity model generated by our refraction-tomography algorithm, and differences between the inverted velocities and the true velocities. The finally inverted model is obtained at the 70th iteration. As usual with the steepestdescent method, we observed a slow-convergence rate. The con- vergence rate can be accelerated by using other methods, such as the conjugate-gradient method. The velocity parameter is updated by equation 23 , and we use a constant value of 100 for the scale factor $\beta$. From Figure 4, we note that the shallow part of the inverted velocity model is comparable to the true model. In Figure 5, we show traveltimes of the inverted velocity model at the 70th iteration, and the history of rms errors. For comparison, we also display traveltimes of the true- and initial-velocity models. The traveltimes of the inverted-velocity model are consistent with those of the true-velocity models.

To evaluate whether the refraction tomography algorithm can be applied successfully to a complicated model, we choose the Marmousi-2 model (Martin et al., 2002) with a grid interval of $20 \mathrm{~m}$ (e.g., Figure 6). In Figure 7, we display first-arrival traveltimes computed with the damped monochromatic wavefield for the Marmousi-2 model when a source is located at $0.1 \mathrm{~km}, 4 \mathrm{~km}$, $8 \mathrm{~km}, 12 \mathrm{~km}$, and $16.9 \mathrm{~km}$. The first-arrival traveltimes are calculated with a damping factor of 62.83 at $0.05 \mathrm{~Hz}$. For convenience, we use the first-arrival traveltimes computed by the damped-wave equation for field data. For the initial model, we also take a velocity model where the velocity increases linearly from $1.5 \mathrm{~km} / \mathrm{s}$ to $4.5 \mathrm{~km} / \mathrm{s}$ with respect to depth. Figure 8 shows the initial model used for refraction tomography, the inverted velocity model pro-
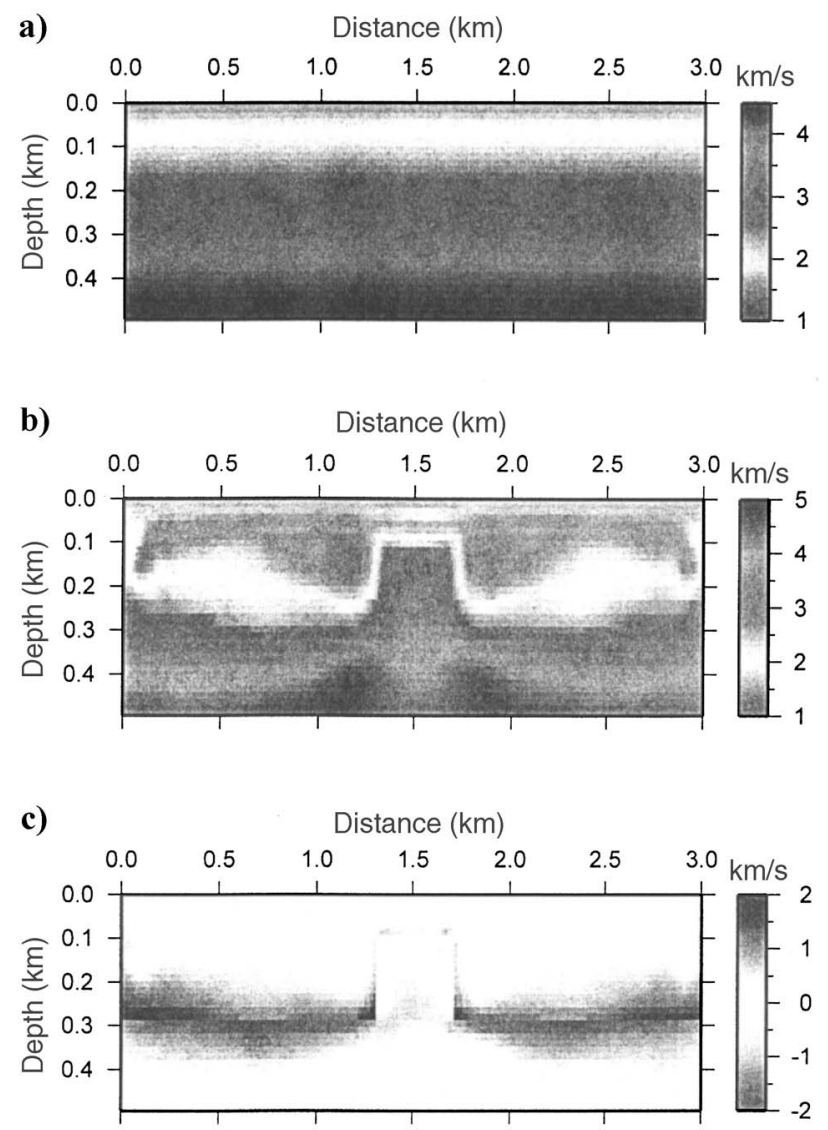

Figure 4. Numerical examples of our refraction-tomography inversion for the block-anomaly model: (a) the initial model, (b) the inverted velocity structure, and (c) differences between inverted and true velocities. 
duced by refraction tomography at the 50th iteration, and discrepancies between the inverted velocities and the true velocities. Figure 9a shows traveltimes calculated for the true Marmousi-2 model, the initial model, and the 50th inverted velocity model. While traveltimes for the initial velocity model are different from those of the true model, traveltimes for the inverted velocity model are very close to those of the true model. Figure $9 \mathrm{~b}$ describes the history of rms errors of the inversion results for the Marmousi-2 model.

We compare our results for the Marmousi-2 model with those of a conventional ray-tracing refraction tomography. In ray-tracing refraction tomography, we apply a simultaneous iterative reconstruction technique (SIRT) (Dines and Lytle, 1979), as Pyun et al. (2005) did. In Figure 10, we display inversion results generated by SIRT at the 25th iteration, and differences between the SIRT inverted model and the true velocity model. By comparing Figure 8 with Figure 10, we note that SIRT gives comparable results to

a)

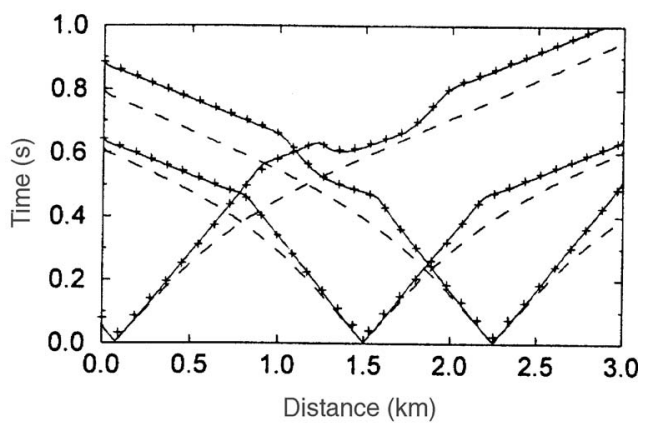

b)

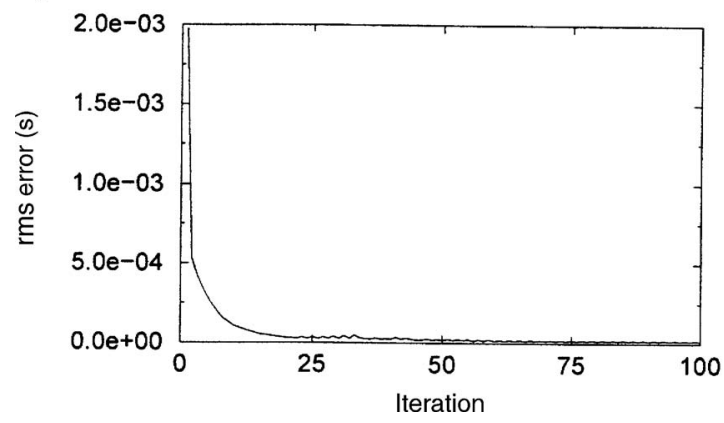

Figure 5. (a) Traveltime curves of the true block-anomaly velocity model (solid lines), the initial model (dashed lines), and the 70th inverted velocity model (plus symbols) when a source is located at $0.075 \mathrm{~km}, 1.5 \mathrm{~km}$, and $2.25 \mathrm{~km}$, and (b) the history of rms errors of the inversion results for the block-anomaly model.

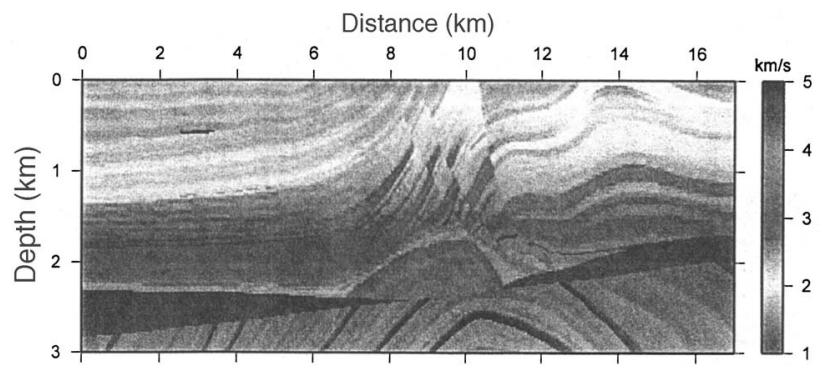

Figure 6. The geometry of the Marmousi-2 model. those for the shallow structure. However, for the deeper part, especially the wedge and anticline structures, SIRT results are not as good as ours (compare discrepancies in Figures 8c and 10b).

Following Pyun et al.'s (2005) approach, we use the velocity structures obtained by refraction tomography for the initial model in Kirchhoff prestack depth migration. For comparison, we also

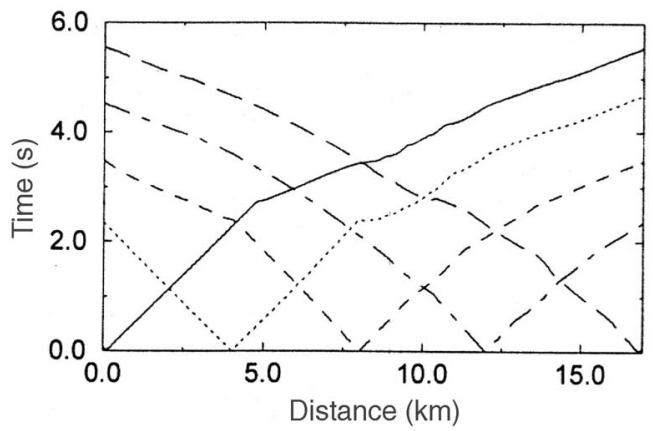

Figure 7. First-arrival traveltimes computed by $0.05-\mathrm{Hz}$ damped monochromatic wavefields for the Marmousi-2 model when a source is located at $0.1 \mathrm{~km}, 4 \mathrm{~km}, 8 \mathrm{~km}, 12 \mathrm{~km}$, and $16.9 \mathrm{~km}$.

a)

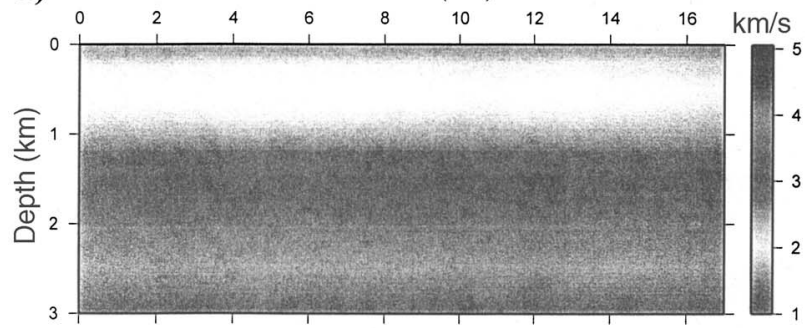

b) Distance $(\mathrm{km})$
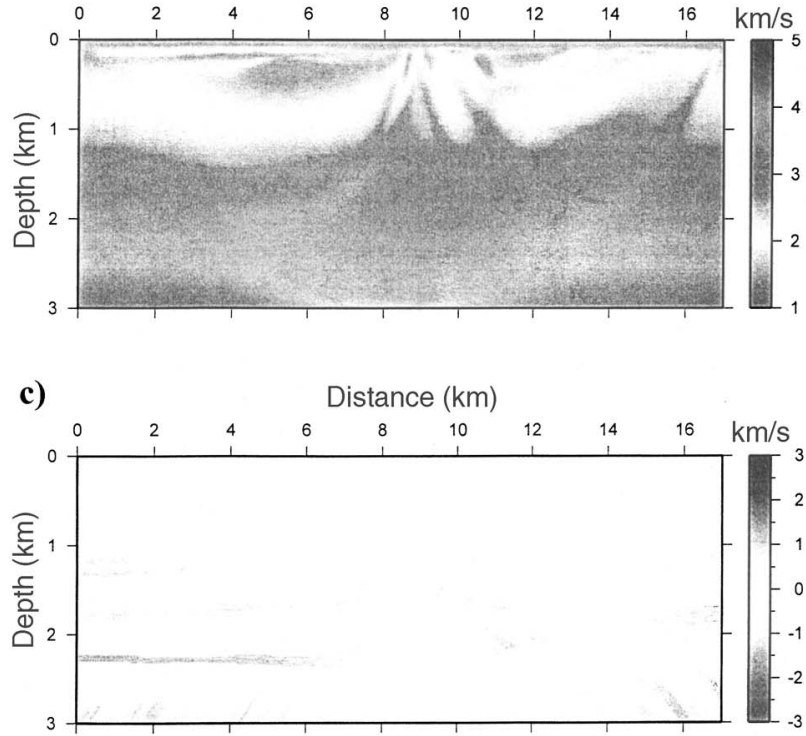

Figure 8. Numerical examples of our refraction-tomography inversion for the Marmousi-2 model, (a) the initial model, (b) the 50th inverted velocity structure, and (c) differences between the inverted velocities and the true velocities. 
a)
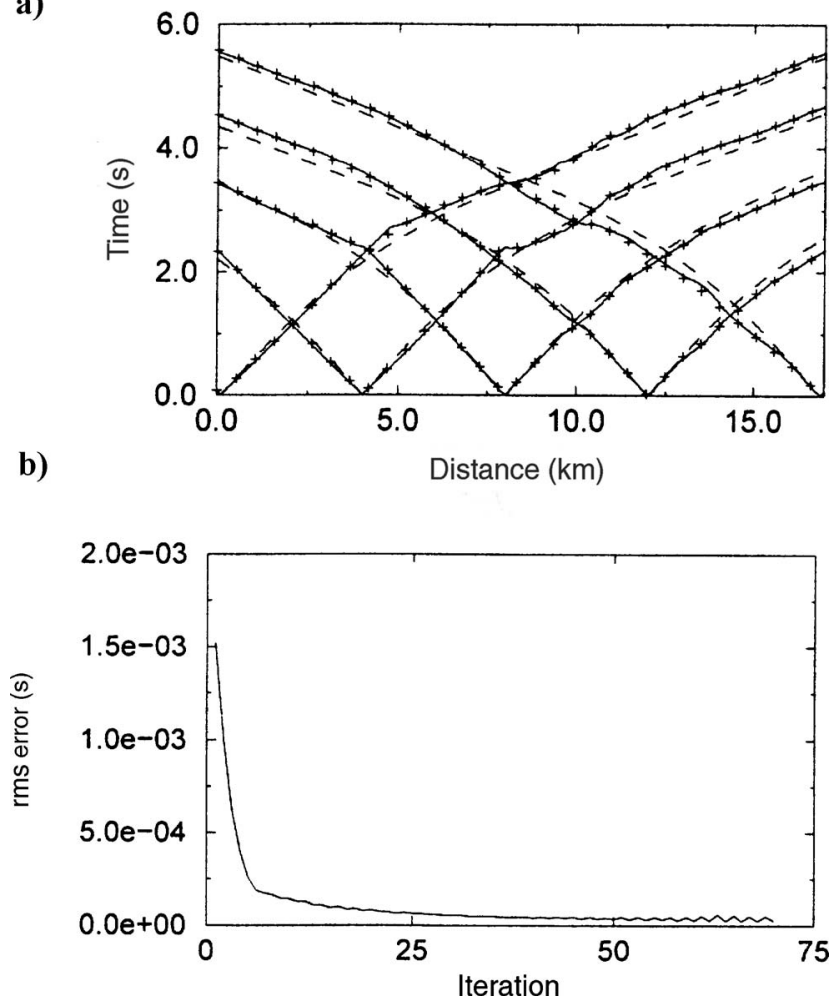

Figure 9. (a) Traveltime curves of the true Marmousi-2 model (solid lines), the initial model (dashed lines), and the 50th inverted velocity model (plus symbols), when a source is located at $0.1 \mathrm{~km}$, $4 \mathrm{~km}, 8 \mathrm{~km}, 12 \mathrm{~km}$, and $16.9 \mathrm{~km}$, and (b) the history of rms errors of the inversion results for the Marmousi-2 model.

a) Distance $(\mathrm{km})$

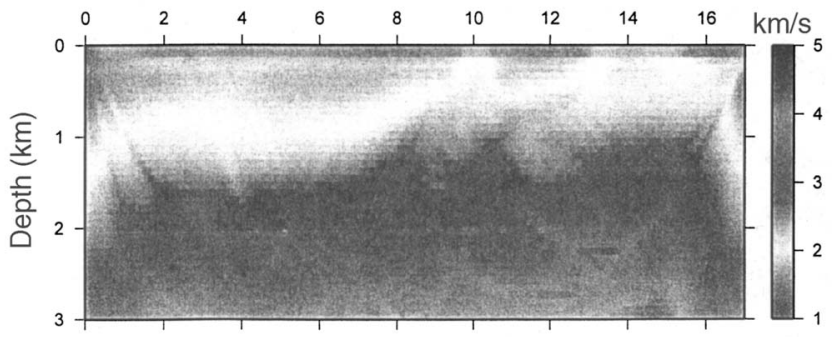

b) $\quad$ Distance $(\mathrm{km})$

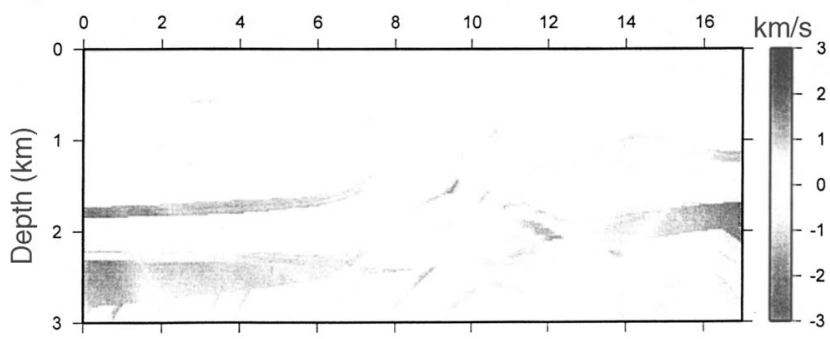

Figure 10. Numerical examples generated by SIRT for the Marmousi-2 model: (a) velocity structures inverted at the 25th iteration, and (b) differences between the inverted velocities and the true velocities. perform Kirchhoff migration using the linearly increasing velocity model and the true-velocity model. For the Kirchhoff migration, we interpolate the velocity structures so that the grid interval is $8 \mathrm{~m}$ and use the most energetic traveltimes synthesized by the finite-difference method (Shin et al., 2003b). Figures 11a-11c, show, respectively, Kirchhoff migration images calculated by using the true-velocity structure, the linearly increasing velocity structure, and the inverted-velocity structure for the Marmousi-2 model. We also display Kirchhoff migration images generated by the SIRT inverted model. In Figure 11, we note that the image generated by our estimated model is comparable to those from the true velocity and SIRT velocity models, and much better than that of the linearly increasing velocity model.
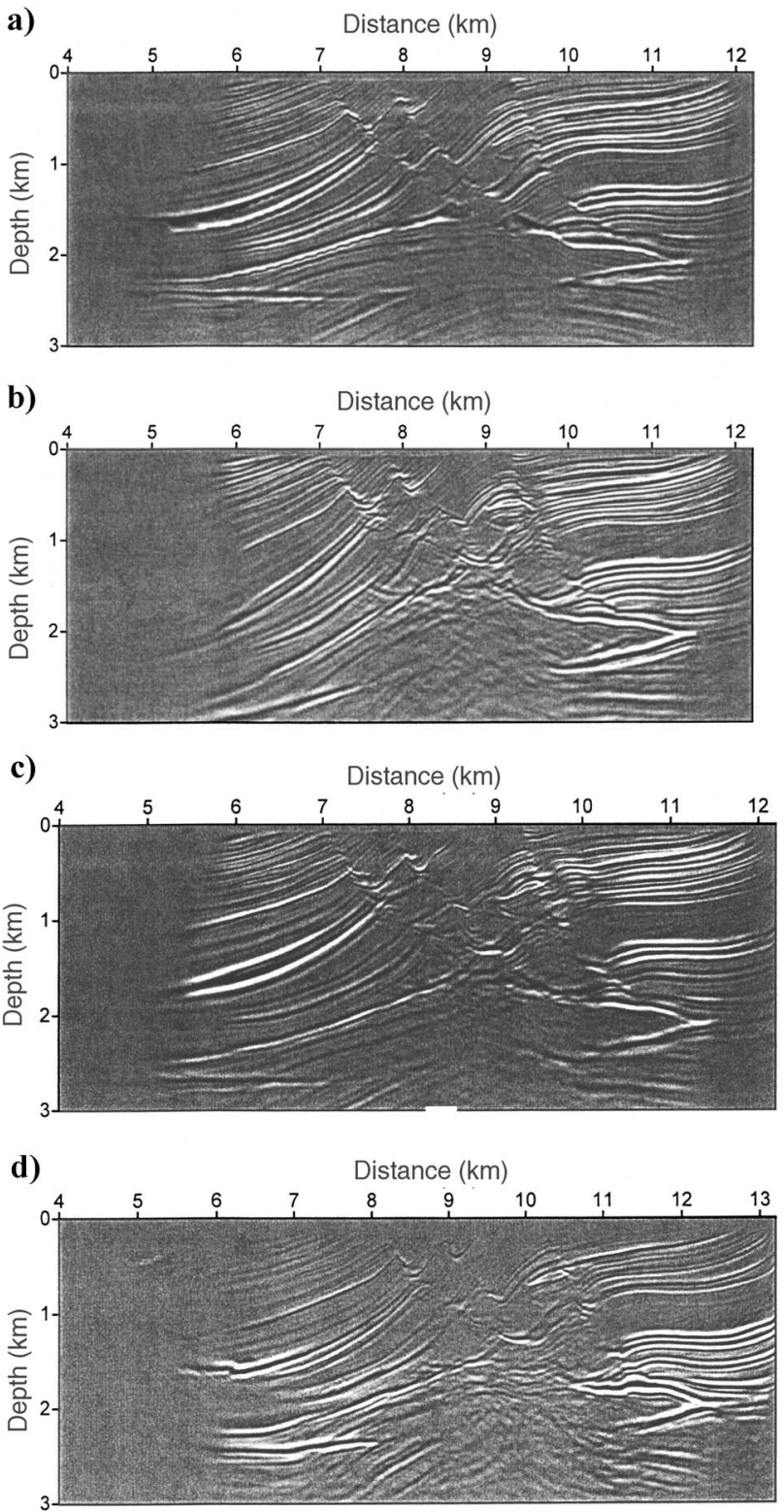

Figure 11. Kirchhoff migration images generated by (a) the true velocity, (b) the linearly increasing velocity, (c) our inverted velocity, and (d) SIRT inverted velocity structure for the Marmousi-2 model. 


\section{CONCLUSIONS}

The introduction of a complex frequency in the wave equation allows us to compute a wavefield consisting largely of a single pulse at the first-arrival time rather than a complex wavetrain following the (possibly small amplitude) first arrival. Taking the natural logarithm of this damped wavefield computed at a single, sufficiently low frequency, we directly extract the phase (and thus the time) of the first arrival. Choosing a very low frequency is necessary to produce unwrapped phase.

Based on the symmetry of the numerical Green's function (or the adjoint state) of the damped-wave equation, we construct a wave-based, refraction-tomography algorithm, whose numerical structure is exactly the same as that of waveform inversion or reverse-time migration, except that we solve a damped-wave equation. In order to compute the steepest-descent direction in our algorithm, we (1) divide the phase differences between field-data and forward modeled-data first arrivals by a modeled wavefield at the surface, (2) back-propagate the divided phase differences, and (3) compute the steepest-descent direction by taking the scalar product of the back-propagated differences and the damped virtual source. This indirect computation of the steepest-descent direction allows us to bypass the burdensome computation of Fréchet derivatives.

The block-anomaly model demonstrated that traveltimes computed from damped wavefields at a single frequency are comparable to traveltimes picked on synthetic data. In order to examine the feasibility of using our tomography algorithm on complicated and high-velocity-contrast models, we applied it to the Marmousi2 model, and then successfully used the inverted-velocity model for building an initial-velocity model for prestack Kirchhoff migration. By comparing the velocity structure generated by our tomography algorithm with that of the ray-based tomography algorithm using SIRT, we demonstrated that our method can yield more reliable results.

\section{ACKNOWLEDGMENTS}

This work was financially supported by grant numbers PE93300 and PM31600 from Korea Ocean Research and Development Institute, National Research Laboratory Project of Ministry of Science and Technology, and Brain Korea 21 project of the Ministry of Education.

\section{APPENDIX A}

\section{DAMPING FACTOR}

Since the damping factor is the imaginary term of the complex angular frequency $\left(\omega^{*}=\omega+i \alpha\right)$, the optimal value of the damping factor can be determined by a numerical dispersion relationship for a damped wave equation expressed as

$$
k^{2}=\frac{\omega^{* 2}}{v^{2}},
$$

which is obtained by replacing the real angular frequency by the complex angular frequency in a general dispersion relationship. When we solve a monochromatic damped wave equation, we use a very low frequency, such as $0.1 \mathrm{~Hz}$ or $0.05 \mathrm{~Hz}$, in order to get unwrapped phases, which enables us to approximate $\omega^{* 2}=\omega^{2}+\alpha^{2}$ $\approx \alpha^{2}(\omega \ll \alpha)$. Then equation A-1 can be rewritten as

$$
k^{2}=\frac{\alpha^{2}}{v^{2}},
$$

which is similar to a dispersion relationship of the Laplacetransformed wave equation in SWEET [see Appendix C in Shin et al. (2002)]. As Shin et al. (2002) did, we can also determine the optimal value of damping factor by using

$$
\begin{gathered}
\alpha^{2}=k^{2} v^{2}, \\
\alpha=k v=\frac{2 \pi}{\lambda} v, \\
\alpha_{\text {optimum }}=\frac{2 \pi}{G \Delta} v_{\text {ave }},
\end{gathered}
$$

where $v_{\text {ave }}$ is the average velocity of a given model, $\Delta$ is the spatial grid interval, and $G$ is the number of grid points per wavelength. The number of grid points per wavelength $G$ is determined by the numerical dispersion relationship (Jo et al., 1996). In our algorithm, since we use the nine-point finite-difference operator suggested by Jo et al. (1996), we choose a value of five for $G$, in order to bound the errors within $1 \%$. If we were to use another numeri cal method, such as the finite-element method, we would need to choose different values for $G$. When $G=5, \Delta=20 \mathrm{~m}$, and $v_{\text {ave }}$ $=2500 \mathrm{~m}$, the optimal damping factor is about 157 . Figure A-1 shows numerical dispersion curves computed for $\alpha=150$. In Figure A-1, we also see that numerical dispersion curve errors are nearly bounded within $1 \%$, with a grid interval of $20 \mathrm{~m}$.

In practice, we use a smaller damping factor than that determined by equation A-5, in order to avoid numerical overflow caused by the 32-bit double precision limit. In our experience, however, a too small damping factor retards the first-arrival traveltimes.

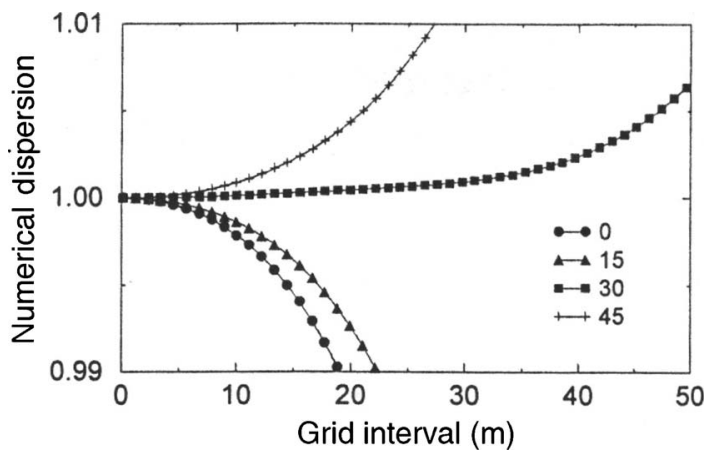

Figure A-1. Numerical dispersion curves when $\alpha=150$ and $v_{\text {ave }}$ $=2500 \mathrm{~m}$, where $0,15,30$, and 45 indicate the propagation angle with respect to $x$ axis. 


\section{APPENDIX B}

\section{AN OPTIMAL FREQUENCY}

In our tomography algorithm, we need unwrapped phases, which are generally obtained by choosing low frequencies (Mora, 1989). In the damped frequency-domain modeling for our inversion algorithm, the optimal frequency for preventing cycle skipping is

$$
f_{\text {optimum }} \leq \frac{1}{t_{\max }}
$$

where $t_{\max }$ is the maximum recording time, which is chosen to be the maximum possible traveltimes of refracted waves in a given velocity model.

\section{APPENDIX C COMPARISON}

We compare our wave-based tomography method with the method suggested by Schuster and Quintus-Bosz (1993). In the method of Schuster and Quintus-Bosz (1993), the objective function is expressed as

$$
E=\frac{1}{2} \sum_{s} \sum_{r} \sum_{\omega} \tilde{R}_{r s}(\omega)\left(\theta_{r s}^{\mathrm{cal}}-\theta_{r s}^{\mathrm{obs}}\right)^{2}
$$

with

$$
\theta_{r s}^{\mathrm{cal}}(\omega)=\operatorname{Im}\left\{\ln \widetilde{u}_{r s}^{\mathrm{cal}}(\omega)\right\}
$$

where $\widetilde{R}_{r s}(\omega)$ is the weighting function, $\theta_{r s}^{\text {cal }}$ and $\theta_{r s}^{\text {obs }}$ are the calculated and observed phases, and $r$ and $s$ indicate the receiver and source positions. The calculated phase $\theta_{r s}^{\text {cal }}(\omega)$ is computed from the imaginary part of the logarithm of the first arrival $\widetilde{u}_{r s}^{\text {cal }}(\omega)$, which is extracted by a time window.

In their method, the gradient with respect to slowness $s(\mathbf{x})$ can be computed by

$$
\frac{\partial E}{\partial s(\mathbf{x})}=\sum_{s} \sum_{r} \sum_{\omega} \widetilde{R}_{r s}(\omega)\left[\theta_{r s}^{\mathrm{cal}}(\omega)-\theta_{r s}^{\mathrm{obs}}(\omega)\right] \frac{\partial \theta_{r s}^{\mathrm{cal}}(\omega)}{\partial s(\mathbf{x})}
$$

with

$$
\frac{\partial \theta_{r s}^{\mathrm{cal}}}{\partial s(\mathbf{x})}=\operatorname{Im}\left\{\frac{\partial\left[\ln \widetilde{u}_{r s}^{\mathrm{cal}}(\omega)\right]}{\partial s(\mathbf{x})}\right\}=\frac{1}{\left|\widetilde{u}_{r s}^{\mathrm{cal}}\right|}\left|\frac{\partial \widetilde{u}_{r s}^{\mathrm{cal}}}{\partial s(\mathbf{x})}\right| \sin \left(\theta_{r s}^{\prime}-\theta_{r s}^{\mathrm{cal}}\right),
$$

where $\theta_{r s}^{\prime}$ is the phase of the Fréchet derivative $\partial \widetilde{u}_{r s}^{\mathrm{cal}} / \partial s(\mathbf{x})$, which is computed by a back-propagation algorithm.
By comparing equations C-3 and C-4 with equation 21, we note that Schuster and Quintus-Bosz (1993) use wrapped phases given by $\left[\sin \left(\theta_{r s}^{\prime}-\theta_{r s}^{\text {cal }}\right)\right]$, whereas we apply unwrapped phases obtained by choosing a low-frequency wavefield. In addition, our method employs only a single-frequency damped wavefield, but Schuster and Quintus-Bosz (1993) apply banded frequencies, permitting our method to be more efficient than that of Schuster and Quintus-Bosz (1993).

\section{REFERENCES}

Aki, K., and P. G. Richards, 1980, Quantitative seismology: Theory and methods: W. H. Freeman and Company.

Dines, K. A., and R. J. Lytle, 1979, Computerized geophysical tomography: Proceedings of the Institute of Electrical and Electronics Engineers, Inc., 67, 1065-1073.

Docherty, P., 1992, Solving for the thickness and velocity of the weathering layer using 2-D refraction tomography: Geophysics, 57, 1307-1318.

Gauthier, O., J. Virieux, and A. Tarantola, 1986, Two-dimensional nonlinear inversion of seismic waveform: Numerical results: Geophysics, 51 1387-1403.

Hampson, D., and B. Russell, 1984, First-break interpretation using generalized linear inversion: 54th Annual International Meeting, SEG, Expanded Abstracts, 532-534.

Jo, C. H., C. Shin, and J. H. Suh, 1996, An optimal 9-point, finitedifference, frequency-space, 2-D scalar wave extrapolator: Geophysics, 61, 529-537.

Lailly, P., 1983, The seismic inverse problem as a sequence of before stack migrations, in J. B. Bednar, R. Redner, E. Robbinson, and A. Weglein, eds., Conference on inverse scattering: Theory and application: Society for Industrial and Applied Mathematics.

Luo, Y., and G. T. Schuster, 1990, Wave equation traveltime inversion: 60th Annual International Meeting, SEG, Expanded Abstracts, 12071210 .

- 1991, Wave equation traveltime inversion: Geophysics, 56, 645653.

Marfurt, K. J., 1984, Accuracy of finite-difference and finite-element modeling in the scalar and elastic wave equations: Geophysics, 49, 533-549.

Martin, G. S., K. J. Marfurt, and S. Larsen, 2002, Marmousi-2: An updated model for the investigation of AVO in structurally complex areas: 72nd Annual International Meeting, SEG, Expanded Abstracts, 1979-1982.

Mora, P., 1989, Inversion = migration + tomography: Geophysics, 54, 1575-1586.

Pratt, R. G., C. Shin, and G. J. Hicks, 1998, Gauss-Newton and full Newton methods in frequency domain seismic waveform inversion: Geophysical Journal International, 133, 341-362.

Pyun, S., C. Shin, D.-J. Min, and T. Ha, 2005, Refraction traveltime tomography using damped monochromatic wavefield: Geophysics, 70, U1U7.

Scales, J. A., P. Docherty, and A. Gersztenkorn, 1990, Regularisation of nonlinear inverse problems: Imaging the near-surface weathering layer: Inverse Problems, 6, 115-131.

Schneider, W. A., and S.-Y. Kuo, 1985, Refraction modeling for static corrections: 54th Annual International Meeting, SEG, Expanded Abstracts, 295-299.

Schuster, G. T., and A. Quintus-Bosz, 1993, Wavepath eikonal traveltime inversion: Theory: Geophysics, 58, 1314-1323.

Shin, C., S. Ko, W. Kim, D.-J. Min, D. Yang, K. J. Marfurt, S. Shin, and K. Yoon, 2003a, Traveltime calculations from frequency domain downward continuation algorithms: Geophysics, 68, 1380-1388.

Shin, C., S. Ko, K. J. Marfurt, and D. Yang, 2003b, Wave equation calculation of most energetic traveltimes and amplitudes for Kirchhoff prestack migration: Geophysics, 68, 2040-2042.

Shin, C., D.-J. Min, K. J. Marfurt, H. Y. Lim, D. Yang, Y. Cha, S. Ko, K. Yoon, T. Ha, and S. Hong, 2002, Traveltime and amplitude calculations using the damped wave solution: Geophysics, 67, 1637-1647.

Stefani, J. P., 1995, Turning-ray tomography: Geophysics, 60, 1917-1929.

Tarantola, A., 1984, Inversion of seismic reflection data in the acoustic approximation: Geophysics, 49, 1259-1266.

Vasco, D. W., J. E. Peterson, Jr., and E. L. Majer, 1995, Beyond ray tomography: Wavepaths and Fresnel volumes: Geophysics, 60, 1790-1804.

White, D. J., 1989, Two-dimensional seismic refraction tomography: Geophysical Journal, 97, 223-245.

Woodward, M. J., 1992, Wave-equation tomography: Geophysics, 57, 1526.

Woodward, M. J., and F. Rocca, 1988, Wave-equation tomography: 58th Annual International Meeting, SEG, Expanded Abstracts, 1232-1235.

Zelt, C. A., and P. J. Barton, 1998, Three-dimensional seismic refraction to- 
mography: A comparison of two methods applied to data from the Faeroe Basin, Journal of Geophysical Research, 103, 7187-7210.

Zhang, J., and M. N. Toksöz, 1998, Nonlinear refraction traveltime tomography: Geophysics, 63, 1726-1737.
Zhu, X., and G. A. McMechan, 1989, Estimation of a two-dimensional seismic compressional-wave velocity distribution by iterative tomographic imaging: International Journal of Imaging Systems and Technology, 1, 13-17. 